\title{
Behavioural analysis of a nonionic detergent in the kraft pulp washing process using cryo-time-of-flight secondary ion mass spectrometry and cryo-scanning electron microscopy
}

\author{
Katsuhiro Tokugawa $^{1} \cdot$ Dan Aoki $^{1} \cdot$ Ryutaro Asai $^{1} \cdot$ Yasuyuki Matsushita $^{1} \cdot$ \\ Masao Ishiguro $^{2} \cdot$ Yasufumi Noda $^{2}$ Kazuhiko Fukushima ${ }^{1}$
}

Received: 11 November 2016 / Accepted: 4 February 2017 / Published online: 14 March 2017

(C) The Japan Wood Research Society 2017

\begin{abstract}
In order to understand the kraft pulp decolouring mechanism on using a nonionic detergent, the pulp washing process and the resulting pulp handsheets were investigated by examining the brightness, kappa number, thioacidolysis product yield, and dewatering efficiency in the pressing sheet making process. The pulp decolouring could be attributed to a decrease in the lignin content and an improvement in the dewatering efficiency. Furthermore, the detergent distribution in the aqueous pulp suspension obtained during the pulp washing process was visualised using cryo-time-of-flight secondary ion mass spectrometry/ scanning electron microscopy (cryo-TOF-SIMS/SEM). The detergent was clearly observed at the transverse surface of the pulp fibre cell wall and was also detected in the lumen of the fibres, suggesting the permeation of the detergent into the pulp fibre cell wall. Based on these results, the pulp decolouring mechanism can be proposed as follows: the detergent permeates into the pulp fibre cell wall and promotes the solution-exchange between the inside and the outside parts of the fibre cell wall, finally washing away the chromophoric substances such as lignin and its degradation products owing to the enhanced dewatering efficiency.
\end{abstract}

Keywords Cryo-TOF-SIMS $\cdot$ Imaging mass spectrometry $\cdot$ Kraft pulping $\cdot$ Detergent $\cdot$ Lignin

Dan Aoki

daoki@agr.nagoya-u.ac.jp

1 Graduate School of Bioagricultural Sciences, Nagoya University, Furo-cho, Chikusa-ku, Nagoya, Aichi 464-8601, Japan

2 Lion Specialty Chemicals Co., Ltd., Honjo, Sumida-ku, Tokyo 130-8644, Japan

\section{Introduction}

Paper making is one of the most developed methods for the usage of sustainable wood biomass. Although the paper making industry has been growing globally for a long time, the process still needs to be improved, such as in terms of reducing the chemical reagent costs, industrial byproducts [1], and high water consumption [2]. The bleaching process of the pulp has attracted significant attention for reducing the release of chemical and energy waste to the environment. In a conventional bleaching process, chromophores such as lignin and its degradation products containing conjugated double bonds that are formed via oxidation during the pulping process [3-6] are degraded with chlorine reagents such as sodium hypochlorite and chlorine dioxide, resulting in the production of toxic chlorinated compounds [7].

Several studies focusing on improving the bleaching process efficiency have been conducted. Chlorine free sequences $[8,9]$ do not use chlorine reagents; nevertheless, the alternative chemicals used in the process such as hydrogen peroxide, oxygen, and ozone are high energy cost reagents. The enzymatic bio-bleaching process has been studied with an aim to decrease the chemical and energy load to the environment [10-12]. The use of nonionic surfactant is another interesting approach for this purpose. Studies investigating the effect of surfactant addition to the digestion process [13] or the delignification process by oxygen [14] were previously carried out, providing positive results for the paper making process.

In contrast to the bleaching process, a few studies have focused on the washing process. After performing the kraft pulping of the wood chip, the pulp suspension undergoes a washing process followed by a bleaching process. Hence, employing an effective washing process of the pulp using a 
detergent should be a promising approach for reducing the bleaching cost. Although a previous study [15] reported an increment in the pulp brightness with detergent addition in the pulp washing process, the detailed mechanism for this is not clear. A deep understanding of the mechanism involved in the process might help us to design an improved pulping process that produces less chemical and energy load to the environment.

In order to understand the behaviour of a specific chemical in a heterogeneous reaction system, the positional information is important. The time-of-flight secondary ion mass spectrometry (TOF-SIMS) imaging technique is a powerful tool for visualising the localisation of target chemical species at the sample surface [16-19]. Recently, we developed a cryo-TOF-SIMS/scanning electron microscopy (SEM) system to analyse freeze-fixed samples and reported the applicability of the system in visualising water-soluble chemicals [20-22]. In a previous study, the adsorption behaviour of the polycationic additive of poly(dimethyldiallylammonium chloride) to pulp fibres in freeze-fixed pulp suspension was successfully visualised [21].

In this study, for further understanding the kraft pulp decolouring mechanism on using a nonionic detergent, the pulp washing process and the resulting pulp handsheets are investigated by examining the brightness, kappa number, thioacidolysis product yield, and dewatering efficiency in the pressing sheet making process. Kraft pulping is carried out using Cryptomeria japonica D. Don wood chip and the obtained pulp are washed with water with or without using the nonionic surfactant, polyoxyalkylene alkyl ether. Paper handsheets are prepared from the pulp, and their brightness, kappa number, thioacidolysis product yield, and water content are evaluated. To visualise the distribution of the detergent in the aqueous suspension during the pulp washing process, a portion of the pulp suspension is frozen using liquid nitrogen. The frozen sample is cut using a sliding microtome, and the transverse surface of the sample is analysed using cryo-TOF-SIMS/SEM. The detergent is detected at the transverse sections of the pulp fibre cell walls and the lumen region of the fibres. Consequently, it can be assumed that the detergent promoted the solutionexchange between the inside and the outside parts of the pulp fibres.

\section{Experimental}

\section{Materials}

The nonionic detergent, polyoxyalkylene alkyl ether (SA60, RO- $\left.\left(\mathrm{CH}_{2} \mathrm{CH}_{2} \mathrm{O}\right)_{\mathrm{n}}-\left(\mathrm{CH}_{2} \mathrm{CH}\left(\mathrm{CH}_{3}\right) \mathrm{O}\right)_{\mathrm{m}}-\mathrm{H}\right)$, was kindly supplied by Lion Specialty Chemicals Co., Ltd. Japan. Kraft pulp samples were obtained by digesting $500 \mathrm{~g}$ wood chip of $C$. japonica using high-pressure micro reactor (MMS-1000, OM lab-tech Co., Ltd., Japan). Kraft pulping was carried out under the following conditions: heating the sample from room temperature to maximum temperature in 90 min, maintaining at the maximum temperature of $170^{\circ} \mathrm{C}$ for $1 \mathrm{~h}$, using active alkali as $\mathrm{NaOH}$ of $24 \%$, sulphidity of $41 \%$, and water ratio of 5 to the wood chip.

After performing kraft pulping, the obtained pulp was washed with water with or without using the detergent. The preparation method of the pulp samples is summarised in Fig. 1. The pulp samples were washed with excess amounts of water twice at $60^{\circ} \mathrm{C}$ for $10 \mathrm{~min}$. The residual pulp thus obtained is named as \#1-0\%. A part of the \#1-0\% sample was separated into four portions containing ca. $10 \mathrm{~g}$ pulp (dry weight) and washed with $250 \mathrm{~mL}$ water at $60^{\circ} \mathrm{C}$ for $10 \mathrm{~min}$ followed by filtering the sample. The samples were then washed with $250 \mathrm{~mL}$ water containing the detergent in desired concentrations $(0,0.3,1.0$, or $3.0 \mathrm{wt} \%$ to pulp dry weight) at $60^{\circ} \mathrm{C}$ for $10 \mathrm{~min}$, and the residual pulp samples thus obtained are named as \#2-0, \#2-0.3, \#2-1.0, \#2-3.0\%, respectively. A half of each \#2 sample containing ca. $5 \mathrm{~g}$ pulp was washed with excess amounts of water four times at $60^{\circ} \mathrm{C}$ for $10 \mathrm{~min}$. The residual pulp samples thus obtained are named as \#3-0, \#3-0.3, \#3-1.0, and \#3-3.0\%. The pulp samples were pressed at $0.2 \mathrm{MPa}$ for $30 \mathrm{~s}$ and air-dried to obtain handsheets.

\section{Brightness and kappa number}

The brightness and the kappa number of each handsheet were evaluated. The brightness was measured using a spectrophotometric brightness meter (PF-10, NIPPON DENSHOKU INDUSTRIES Co., Ltd, Japan) employing a colour-mate PF software and is expressed with an $L^{*} a^{*} b^{*}$

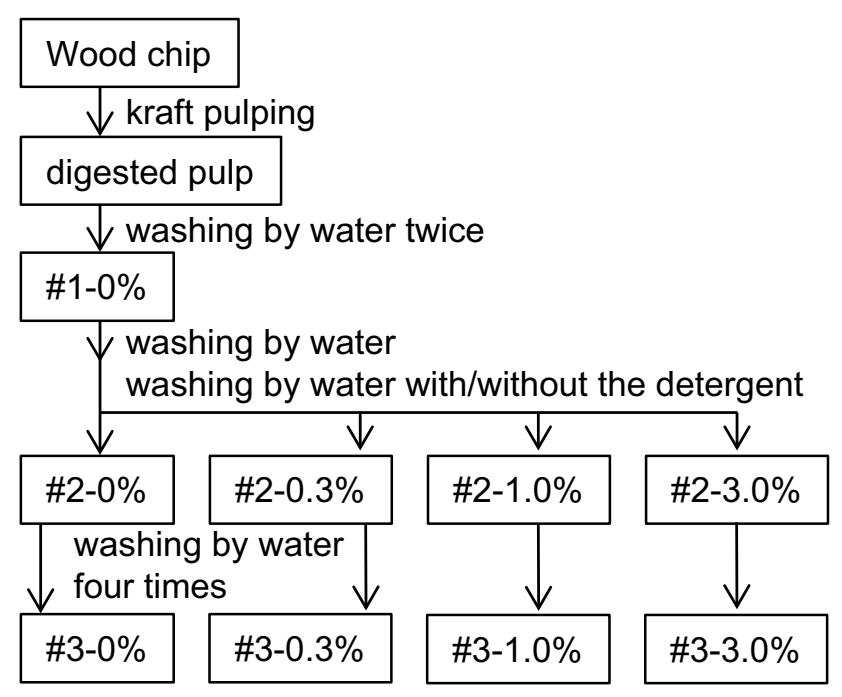

Fig. 1 Preparation of kraft pulp samples 
colour scale. The measurement was conducted for 12 times for each handsheet sample.

The kappa number was measured according to ISO 302:2015 [23]. Before performing the measurements, the pulp samples were chopped using a cutting mill (PULVERISETTE 14, Fritsch, Germany) with a $0.5 \mathrm{~mm}$ mesh and stored in a desiccator with silica gel for several days. The sample was suspended in $400 \mathrm{~mL}$ water, and the reaction was started by adding $50 \mathrm{~mL} 2 \mathrm{M} \mathrm{H}_{2} \mathrm{SO}_{4}$ aqueous solution (aq.) and $50 \mathrm{~mL} 0.02 \mathrm{M} \mathrm{KMnO}_{4}$ aq. at $25^{\circ} \mathrm{C}$. After stirring the solution for $10 \mathrm{~min}$, the reaction was quenched by adding $10 \mathrm{~mL}$ of $1 \mathrm{M} \mathrm{KI}$ aq., and the solution was immediately titrated with $0.2 \mathrm{M} \mathrm{Na}_{2} \mathrm{~S}_{2} \mathrm{O}_{3}$ aq. using $\mathrm{I}_{2}$ as an indicator. The measurements were triplicated for each sample.

\section{Thioacidolysis}

The lignin content in the pulp handsheet samples was evaluated by carrying out thioacidolysis and gas chromatography-mass spectrometry (GC-MS) measurements according to a previously reported protocol [24]. The reaction reagent was prepared by adding $1.25 \mathrm{~mL}$ boron trifluoride diethyl ether complex and $5 \mathrm{~mL}$ ethanethiol into $43.75 \mathrm{~mL}$ anhydrous dioxane. Approximately $50 \mathrm{mg}$ of pulp sample, $0.5 \mathrm{~mL}$ of $10 \mathrm{mg} / \mathrm{mL} n$-docosane/dioxane solution, and $5 \mathrm{~mL}$ of the reaction reagent were mixed in a test tube. The reaction was carried at $100^{\circ} \mathrm{C}$ for $4 \mathrm{~h}$ while shaking the mixture every $20 \mathrm{~min}$. The reaction was quenched by cooling on ice and adding $2.5 \mathrm{~mL} 0.4 \mathrm{M} \mathrm{NaHCO}_{3}$ aq. The $\mathrm{pH}$ of the solution was adjusted to $2-3$ with dilute $\mathrm{HCl}$ aq., and the mixture was extracted using dichloromethane (5 mL each, 3 times), dried over $\mathrm{Na}_{2} \mathrm{SO}_{4}$, and condensed under a reduced pressure at $40{ }^{\circ} \mathrm{C}$. The silylated derivatives of thioacidolysis monomer products were quantified using a GC-MS (TRACE 1300, ITQ 900, Thermo Fisher Scientific K.K, Japan) apparatus equipped with a capillary column $(30 \mathrm{~m} \times 0.32 \mathrm{~mm}$ id, film thickness of $0.25 \mu \mathrm{m}$, $\mathrm{Rxi}^{\circledR}-1 \mathrm{~ms}$; Restek Corporation, US) with a retention factor of 1.5 to $n$-docosane. Each sample was injected at $180^{\circ} \mathrm{C}$, and the temperature was programmed to increase from 180 to $230^{\circ} \mathrm{C}$ at the rate of $2{ }^{\circ} \mathrm{C} \mathrm{min}{ }^{-1}$ and from 230 to $300^{\circ} \mathrm{C}$ at the rate of $15^{\circ} \mathrm{C} \mathrm{min}^{-1}$. The thioacidolysis process was triplicated for each sample, and the GC-MS measurements were carried out twice for each trial. Furthermore, the whole procedure of thioacidolysis and GC-MS measurements was triplicated. In total, 18 values were obtained for each sample, which were used to calculate the average and the standard deviation values for each sample.

\section{Dewatering efficiency}

In order to evaluate the effect of detergent on the dewatering efficiency of the pulp handsheets, the sample \#3-0\% was used for the experiments. Four samples containing $1.0 \mathrm{~g}$ of \#3-0\% were washed with $100 \mathrm{~mL}$ water containing the detergent in desired concentrations $(0,0.3,1.0$, or $3.0 \mathrm{wt} \%$ to pulp) at $60^{\circ} \mathrm{C}$ for $1 \mathrm{~min}$. Handsheets were prepared by filtration and pressing at $0.2 \mathrm{MPa}$ for $30 \mathrm{~s}$. The water content in the handsheets obtained just after pressing was evaluated. The experiment was triplicated.

\section{Cryo-TOF-SIMS/SEM analysis}

Immediately after washing the \#2-0\% and \#2-3.0\% samples, a small portion of the pulp suspension was frozen by dropping it into liquid nitrogen and was stored at $-80^{\circ} \mathrm{C}$ before use. The frozen sample was fixed on the sample holder and cut using sliding microtome at $-30^{\circ} \mathrm{C}$ in a glove box filled with dry and cooled nitrogen gas. The fresh and flat surface of the sample was analysed. The sample holder was transferred to the cryo-TOF-SIMS using a cryo-vacuum transfer shuttle. The details of the cryo-TOF-SIMS/ SEM system have been reported previously [20,21].

Cryo-TOF-SIMS measurements were conducted using a TRIFT III spectrometer (ULVAC-PHI, Inc., Kanagawa, Japan). Positive ion spectra were obtained under the following conditions: using primary ion of $22 \mathrm{keV} \mathrm{Au}_{1}{ }^{+}$ at a current $5 \mathrm{nA}$; raster size of $300 \mu \mathrm{m} \times 300 \mu \mathrm{m}$; pulse width of $1.8 \mathrm{~ns}$ (bunched for spectrum mode) or $13.0 \mathrm{~ns}$ (not bunched for image mode); mass range, $\mathrm{m} / \mathrm{z}$ of $0-1850$; spot size of $1.0 \mu \mathrm{m}$ in image mode; temperature of -120 to $-130^{\circ} \mathrm{C}$; a low-energy pulsed electron gun $(30.0 \mathrm{eV})$ was used for surface charge compensation. Subsequently, the same region was examined using cryo-scanning electron microscopy (cryo-SEM). The conditions employed for the observation were as follows: acceleration voltage of $1.5 \mathrm{kV}$; temperature of $-120^{\circ} \mathrm{C}$; working distance of $10 \mathrm{~mm}$. The overlay image of the selected cryo-TOF-SIMS ion on cryoSEM image was obtained using Photoshop CS5 Extended (Adobe Systems Incorporated).

\section{Results and discussion}

\section{Brightness and kappa number}

The brightness and the kappa number of the pulp \#1, and \#2 samples are summarised in Table 1. On comparing the samples \#1-0, \#2-0, and \#3-0\%, we can find that the brightness of the handsheets increased on washing with water. Furthermore, the samples washed with the detergent (\#20.3 , \#2-1.0, and \#2-3.0\%) exhibited a higher brightness as compared with the sample washed without using the detergent (\#2-0\%). A similar tendency was observed on performing repeated washing with water as indicated by the results obtained for \#3 samples. This result confirmed the 
Table 1 Brightness and Kappa number of pulp sheet samples

\begin{tabular}{lll}
\hline Samples (\%) & Brightness $(\%)$ & Kappa number \\
\hline$\# 1-0$ & $21.9 \pm 0.8$ & $29.6 \pm 0.4$ \\
$\# 2-0$ & $27.9 \pm 0.6$ & $28.3 \pm 0.2$ \\
$\# 2-0.3$ & $30.3 \pm 0.7$ & $27.9 \pm 0.9$ \\
$\# 2-1.0$ & $29.3 \pm 0.5$ & $28.5 \pm 0.5$ \\
$\# 2-3.0$ & $33.2 \pm 0.9$ & $28.3 \pm 0.5$ \\
$\# 3-0$ & $36.8 \pm 0.3$ & - \\
$\# 3-0.3$ & $37.5 \pm 0.3$ & - \\
$\# 3-1.0$ & $38.0 \pm 0.4$ & - \\
$\# 3-3.0$ & $38.5 \pm 0.3$ & - \\
\hline
\end{tabular}

detergent contribution towards the brightness increment of the pulp samples.

The kappa number slightly decreased from $29.6 \pm 0.4$ for \#1-0\% to $28.3 \pm 0.2$ for \#2-0\% on washing with water. However, a significant difference could not be observed with the \#2 samples. The determination of the kappa number is a convenient method for estimating the amount of oxidisable substances such as lignin only by titration. However, in this experiment, the standard deviation might be larger than the difference obtained within the pulp samples.

\section{Lignin content evaluation by thioacidolysis}

As mentioned above, lignin and its derivatives are the major chromophores present in kraft pulp. To evaluate the remaining lignin content in the pulp samples more precisely, thioacidolysis and GC-MS analysis were conducted. In thioacidolysis, the $\beta-O-4$ linkage in lignin is selectively cleaved and a characteristic product of guaiacyl trithioethyl phenylpropane (G-T) can be detected. The evaluated lignin contents are given in Fig. 2 as a percentage to that of \#3-0\%.

As a result of washing with the detergent, the yield of G-T of the pulp samples decreased ( $p<0.05$ in comparison with that of \#3-0\%). A decline of nearly $20 \%$ in

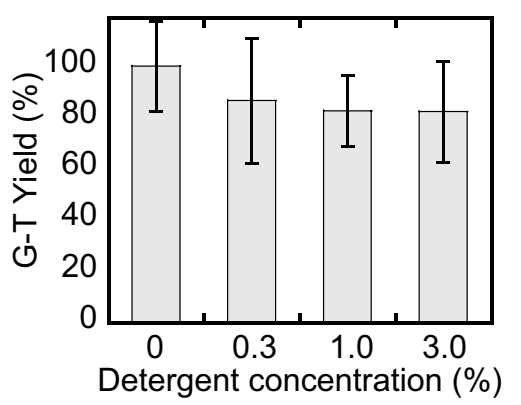

Fig. 2 G-T yield obtained for the \#3 samples evaluated by thioacidolysis and GC-MS measurements average was achieved. The difference between the detergent-washed samples was not significant $(p>0.1)$. A tendency similar to that of the brightness was observed, suggesting the largest decline occurring between \#3-0 and \#3-0.3\% and smaller decline values among the detergentwashed samples. The thioacidolysis results indicated that the detergent-washing enhanced the removal of lignin from the pulp samples.

\section{Dewatering efficiency}

The water content in the pulp handsheets was measured in order to estimate the dewatering efficiency in the handsheet making process $[25,26]$. The evaluated water content of the samples is displayed in Fig. 3. The detergent-washed pulp handsheet samples exhibited lower water content as compared with the sample washed only with water. The result indicated that the detergent enhanced the dewatering efficiency. One of the factors leading to the enhancement could be the reduction of interfacial tension by the detergent. The enhanced dewatering might contribute to the effective washing of the chromophores in the pulp and this might be a reason for the higher brightness obtained. The water content of the sample $0.3 \%$ given in Fig. 3 is ca. $1 \%$ lower than that of the sample $0 \%$. The difference is much smaller than that obtained with the lignin content (ca. 13\%) evaluated by thioacidolysis.

\section{Distribution and the behaviour of the detergent in the pulp washing process}

The distribution of the detergent in the pulp sample during the washing process was examined by analysing the frozen sample using the cryo-TOF-SIMS/SEM system. Cryo-TOF-SIMS spectra of the aqueous solution of the detergent, \#2-0\% suspension, and \#2-3.0\% suspension are shown in Fig. 4. In the region of $m / z$ of 400-800, characteristic secondary ions could be detected in the aqueous solution of the detergent (Fig. 4a). The $\mathrm{m} / \mathrm{z}$ differences of these

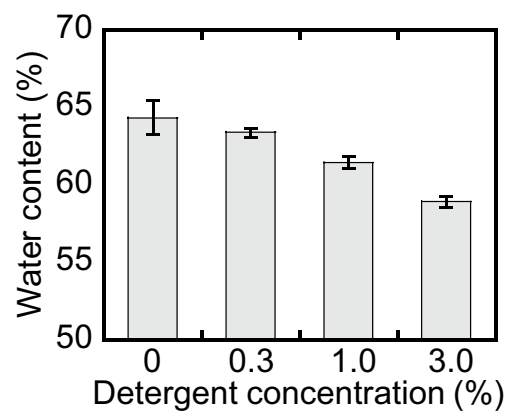

Fig. 3 Water content of the pulp handsheet samples washed with or without the detergent evaluated using \#3-0\% as the starting material 

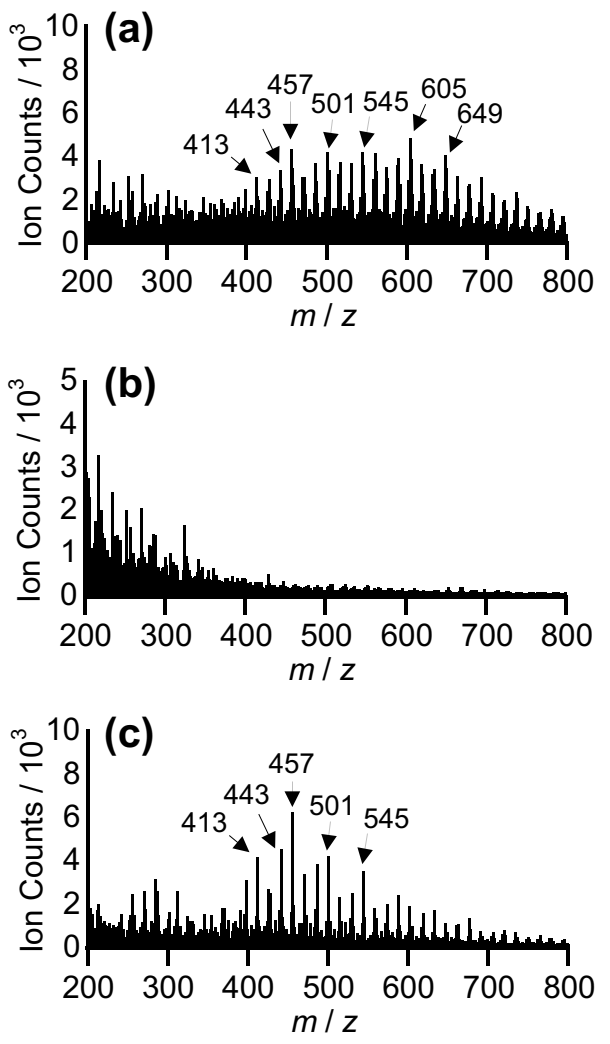

Fig. 4 Cryo-TOF-SIMS spectra of a aqueous solution of the detergent, b \#2-0\%, and c \#2-3.0\%

ions were mainly 44 and 58 , suggesting that they could be attributed to the fragment ions of polyoxyalkylene alkyl ether containing $\mathrm{CH}_{2} \mathrm{CH}_{2} \mathrm{O}(44 \mathrm{Da})$ and $\mathrm{CH}_{2} \mathrm{CH}\left(\mathrm{CH}_{3}\right) \mathrm{O}$ $(58 \mathrm{Da})$ units. These ions were not detected in the waterwashed sample (Fig. 4b), but were clearly detected in the detergent-washing sample (Fig. 4c). Hence, it should be possible to use these ions for visualising the detergent in the pulp suspension. For this purpose, the strongest ion corresponding to $\mathrm{m} / \mathrm{z}$ of 457 was used.

Cryo-TOF-SIMS measurements followed by cryo-SEM examinations were successively conducted to analyse the same surface. The resultant images of the \#2-3.0\% sample are summarised in Fig. 5. As displayed in the cryo-TOFSIMS total ion image (Fig. 5a) and the cryo-SEM image (Fig. 5c), circular transverse sections of the digested pulp fibres, in other words, the cell walls of the fibres could be clearly recognised. The pulp fibres were frozen as a suspension, and they were embedded in the washing water. The circular transverse sections of the pulp fibres exhibited various shapes suggesting that the wood chips were successfully separated into independent fibres and the fibres oriented randomly in the suspension.
Distribution of the detergent is shown in Fig. 5b. To observe the detergent distribution in detail, the $\mathrm{m} / \mathrm{z} 457$ ion image was overlaid on the cryo-SEM image (Fig. 5d). The detergent was mainly detected in the washing water. The detergent was also detected in the lumen region of the pulp fibres. These results indicated the solution exchange that occurring between the inside and the outside parts of the fibre. Furthermore, the detergent could be well detected at the transverse sections of the pulp fibres rather than at the washing water region. Naturally, the solutions of the inside and the outside parts of the fibre cell walls should also be exchanged during the washing process and the solution exchange can be accelerated by the permeation of the detergent into the cell wall. This situation is different with that observed with the case of the polycationic additive of poly(dimethyldiallylammonium chloride), which was detected at the fibre surface but not at the transverse section of the fibre [21]. This difference could be attributed to the nonionic character of the detergent.

\section{Conclusion}

In this study, the kraft pulp decolouring mechanism obtained with the nonionic detergent in the pulp washing process was investigated. The use of the detergent caused an increase in the brightness of the resultant pulp handsheets. The chemical differences of the handsheets were examined by determining the kappa number and by employing thioacidolysis. Although the accuracy of kappa number evaluation was not sufficient to clarify the difference, the thioacidolysis G-T yield examinations indicated the presence of lower lignin contents in the detergent-washed pulp samples. Moreover, the dewatering efficiency of the pulp improved significantly with the detergent addition.

Distribution of the detergent in the aqueous pulp suspension during the washing process was successfully visualised by employing cryo-TOF-SIMS/SEM analysis. The detergent was clearly detected at the transverse sections of the pulp fibre cell walls. The detergent was also detected at the lumen region of the pulp fibres. Hence, the pulp decolouring mechanism occurring with the detergent addition can be proposed as follows: the detergent permeates into the pulp fibre cell wall and promotes the solution-exchange between the inside and the outside parts of the fibre cell wall, finally washing away the chromophoric substances such as lignin and its degradation products owing to the enhanced dewatering efficiency. 
(a) total ion

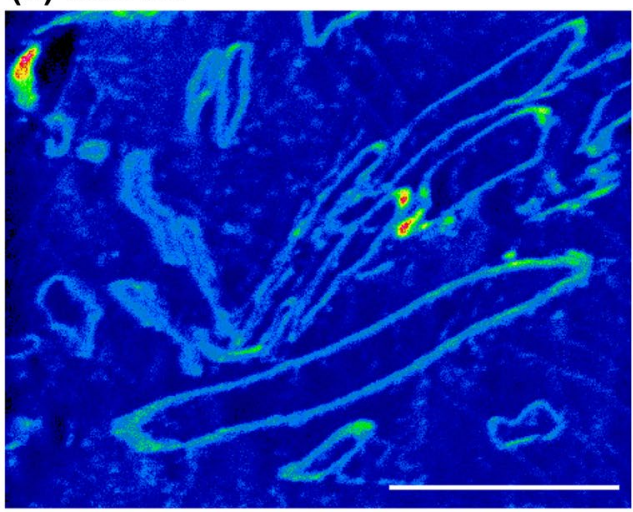

(c) cryo-SEM image

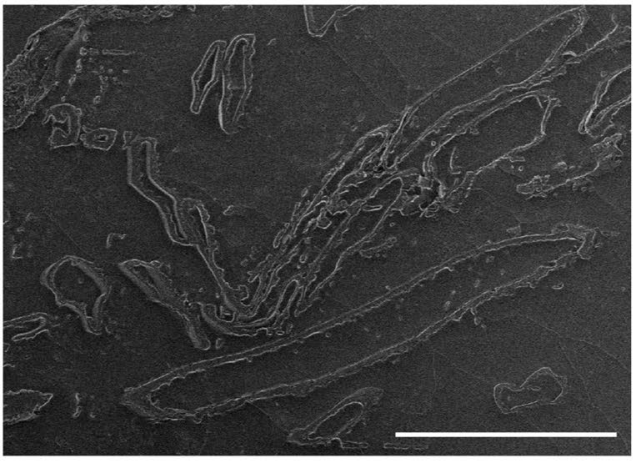

(b) $m / z 457$

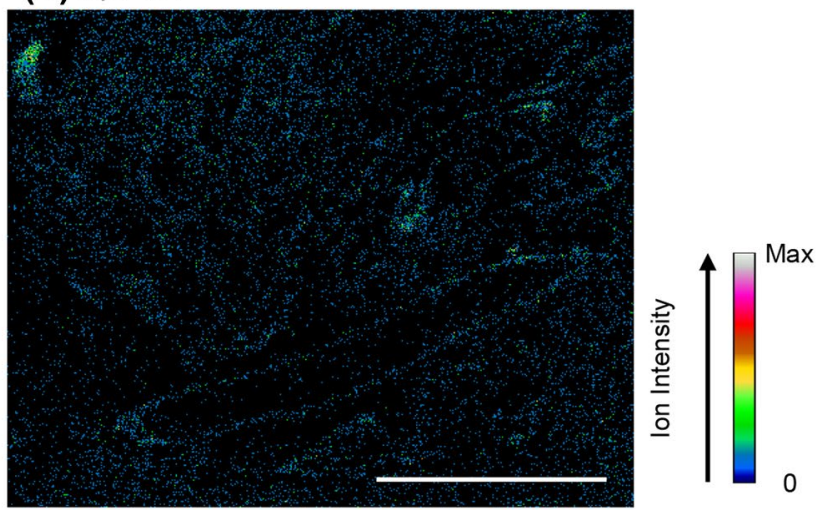

(d) Overlay image of $\mathrm{m} / \mathrm{z} 457$ on cryo-SEM image

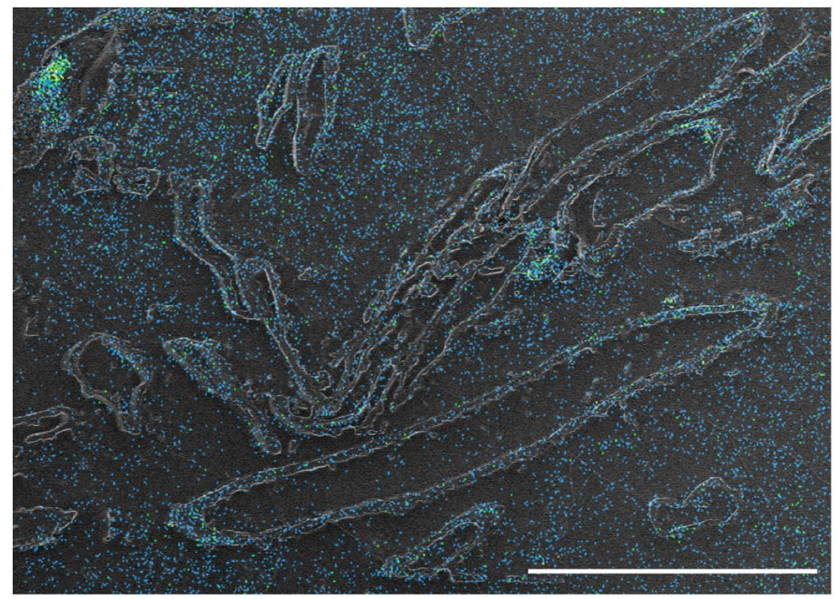

Fig. 5 Images of the transverse surface of the frozen \#2-3.0\%: a cryo-TOF-SIMS total ion image, b cryo-TOF-SIMS $m / z 457$ ion image, $\mathbf{c}$ cryoSEM image, and $\mathbf{d} m / z 457$ ion image overlaid on the cryo-SEM image of the same region. Scale bars correspond to $100 \mu \mathrm{m}$

Acknowledgements This work was supported by Grant-in-Aid for Scientific Research (25252032) from the Ministry of Education, Culture, Sports, Science and Technology (MEXT) of Japan.

\section{References}

1. Ali M, Sreekrishnan TR (2001) Aquatic toxicity from pulp and paper mill effluents: a review. Adv. Environ Res 5:175-196

2. Thompson G, Swain J, Kay M, Forster CF (2001) The treatment of pulp and paper mill effluent: a review. Bioresour Technol 77:275-286

3. Gellerstedt G (2010) Chemistry of pulp bleaching. In: Heitner C, Dimmel DR, Schmidt JA (eds) Lignin and lignans advances in chemistry. CRC Press, London, pp 393-438

4. Gierer J, Lenic J, Norén I, Szabo-Lin I (1974) Lignin chromophores. Part I. Synthesis of chromophores of the 2,4'- and 4,4'-dihydroxystilbene types. Acta Chem Scand B 28:717-729

5. Gierer J, Pettersson I, Szabo-Lin I (1974) Lignin chromophores. Part II. The behaviour of 2,4'- and 4,4'-dihydroxystilbene structures towards oxygen-alkali. Acta Chem Scand B 28:1129-1135
6. Lonsky L, Lonsky W, Kratzl K, Falkehag I (1976) Synthesis and reactions of hydroxylated stilbenes and their possible occurrence as chromophore precursor structures in lignin. Monatsh Chem 107:685-695

7. Kringstad KP, Lindström K (1984) Spent liquors from pulp bleaching. Environ Sci Tech 18:236A-248A

8. Ragnar M, Dahllöf H (2002) ECF bleaching of Eucalypt kraft pulp_-bleaching chemical needs and yellowing characteristics of different sequences. Nord Pulp Pap Res J 17:228-233

9. Roncero MB, Torres AL, Colom JF, Vidal T (2000) Effects of xylanase treatment on fibre morphology in totally chlorine free bleaching (TCF) of Eucalyptus pulp. Process Biochem 36:45-50

10. Camarero S, García O, Vidal T, Colom J, Río JCD, Gutiérrez A, Gras JM, Monje R, Martínez MJ, Martínez ÁT (2004) Efficient bleaching of non-wood high-quality paper pulp using laccasemediator system. Enzym Microb Tech 35:113-120

11. Khandeparkar R, Bhosle NB (2007) Application of thermoalkalophilic xylanase from Arthrobacter sp. MTCC 5214 in biobleaching of kraft pulp. Bioresour Technol 98:897-903

12. Sasaki T, Kajino T, Li B, Sugiyama H, Takahashi H (2001) New pulp biobleaching system involving manganese peroxidase immobilized in a silica support with controlled pore sizes. Appl Environ Microbiol 67:2208-2212 
13. Hamzeh Y, Abyaz A, Niaraki MOM, Abdulkhani A (2009) Application of surfactants as pulping additives in soda pulping of bagasse. BioResources 4:1267-1275

14. Allen L, Schofield M, Faubert M, Bouchard J (2005) Improved deresination during oxygen delignification. Part II: effects of blended surfactant addition. Pulp Pap (Canada) 106:41-43

15. Yamauchi M, Furuya S, Shimoya T, Nikaidoh M, Noda Y, Ushiyama H, Koide M (2009) Detergent for kraft pulp and process for producing kraft pulp with the same. WO2009093615 A1

16. Fardim P, Holmbom B (2005) Origin and surface distribution of anionic groups in different papermaking fibres. Colloid Surface Physicochem Eng Aspect 252:237-242

17. Freire CSR, Silvestre AJD, Neto CP, Gandini A, Fardim P, Holmbom B (2006) Surface characterization by XPS, contact angle measurements and ToF-SIMS of cellulose fibers partially esterified with fatty acids. J Colloid Interface Sci 301:205-209

18. Fardim P, Durán N (2002) Surface chemistry of Eucalyptus wood pulp fibres: effects of chemical pulping. Holzforschung 56:615-622

19. Fardim P, Gustafsson J, Schoultz SV, Peltonen J, Holmbom B (2005) Extractives on fiber surfaces investigated by XPS, ToFSIMS and AFM. Colloid Surface Physicochem Eng Aspect 255:91-103
20. Kuroda K, Fujiwara T, Imai T, Takama R, Saito K, Matsushita Y, Fukushima K (2013) The cryo-TOF-SIMS/SEM system for the analysis of the chemical distribution in freeze-fixed Cryptomeria japonica wood. Surf Interface Anal 45:215-219

21. Masumi T, Matsushita Y, Aoki D, Takama R, Saito K, Kuroda K, Fukushima K (2014) Adsorption behavior of poly(dimethyldiallylammonium chloride) on pulp fiber studied by cryo-timeof-flight secondary ion mass spectrometry and cryo-scanning electron microscopy. Appl Surf Sci 289:155-159

22. Aoki D, Hanaya Y, Akita T, Matsushita Y, Yoshida M, Kuroda K, Yagami S, Takama R, Fukushima K (2016) Distribution of coniferin in freeze-fixed stem of Ginkgo biloba L. by cryo-TOFSIMS/SEM. Sci Rep 6:31525. doi:10.1038/srep31525

23. ISO 302:2015 (2015) Pulps-determination of Kappa number. International Organization for Standardization, Geneva

24. Lapierre C, Pollet B, Monties B, Rolando C (1991) Thioacidolysis of spruce lignin: GC-MS analysis of the main dimers recovered after raney nickel desulphuration. Holzforschung 45:61-68

25. Maloney T, Todorovic A, Paulapuro H (1998) The effect of fiber swelling on press dewatering. Nord Pulp Pap Res J 4:285-291

26. Blomstedt M, Asikainen J, Lähdeniemi A, Ylönen T, Paltakari J, Hakala KT (2010) Effect of xylanase treatment on dewatering properties of birch kraft pulp. BioResources 5:1164-1177 